\title{
Isolated Primary Cardiac Sarcoidosis Presenting as Acute Heart Failure
}

Yoichiro Sugizaki, Hidekazu Tanaka, Junichi Imanishi, Akihide Konishi, Tomoya Yamashita, Toshiro Shinke, Tatsuro Ishida, Hiroya Kawai and Ken-ichi Hirata

\begin{abstract}
A 65-year-old man was referred to our hospital due to an acute onset of dyspnea and persistent fever. Echocardiography revealed an ejection fraction (EF) of $25 \%$ with diffuse severe left ventricular (LV) dysfunction. 18F-fluorodeoxy glucose-positron emission tomography imaging showed significantly increased uptake by the LV and right ventricular walls, indicating active inflammation. The histologic findings of the endomyocardial biopsy specimens indicated the presence of epithelioid cell granuloma. The final diagnosis was thus cardiac sarcoidosis with acute inflammation. Five-months after the initiation of steroid therapy, echocardiography showed an $\mathrm{EF}$ of $50 \%$. This is a rare case in which acute inflammation led to acute heart failure mimicking acute myocarditis.
\end{abstract}

Key words: cardiac sarcoidosis, acute heart failure, echocardiography, myocarditis

(Intern Med 52: 71-74, 2013)

(DOI: 10.2169/internalmedicine.52.8470)

\section{Introduction}

Sarcoidosis is a systemic granulomatous disease of undefined cause involving multiple organs, including the lungs, lymph nodes, eyes, heart and skin. Uneven wall motion abnormalities characterized by the presence of dyskinetic or akinetic segments coexistent with normokinetic segments have been frequently observed in patients with cardiac sarcoidosis. On the other hand, patients with acute heart failure (HF) caused by severely diffuse left ventricular (LV) wall motion abnormalities mimicking acute myocarditis in the development of cardiac sarcoidosis are extremely rare. This case report concerns an acute HF patient with acute inflammation caused by cardiac sarcoidosis who was successfully treated with prednisolone rather than optimal pharmacological therapy for HF.

\section{Case Report}

A 65-year-old man with a history of diabetes mellitus was admitted to a local hospital in August 2011 due to an acute onset of dyspnea and persistent fever. The patient was then referred to our hospital in September 2011 because his acute HF had not improved in spite of having undergone optimal pharmacological therapy. On admission, the patient's blood pressure was $114 / 80 \mathrm{~mm} \mathrm{Hg}$, his heart rate was 112 beats per minute, and his oxygen saturation was $89 \%$ or above on room air. The jugular veins were distended with a prominent $\mathrm{v}$ wave, indicating an elevated right atrial pressure and significant tricuspid regurgitation. Auscultation revealed a third heart sound and a Levine III/VI systolic murmur at the left lower sternal border, while coarse crackles were detected in both lower lung fields. Pitting edema was observed in both legs. The laboratory findings showed a brain natriuretic peptide level of $731 \mathrm{pg} / \mathrm{mL}$ (reference range: $<18.4 \mathrm{pg} / \mathrm{mL}$ ), a C-reactive protein level of $5.66 \mathrm{mg} / \mathrm{dL}$ (reference range: < $0.3 \mathrm{mg} / \mathrm{dL}$ ), a creatine kinase muscle and brain (CKMB) level of $9.0 \mathrm{U} / \mathrm{L}$ (reference range: $<12 \mathrm{U} / \mathrm{L}$ ), an angiotensin converting enzyme level of $9.3 \mathrm{U} / \mathrm{L}$ (reference range: 7.7$29.4 \mathrm{U} / \mathrm{L}$ ), and a serum calcium concentration of $8.0 \mathrm{mg} / \mathrm{dL}$ (reference range: $8.4-9.9 \mathrm{mg} / \mathrm{dL}$ ). A tuberculin skin test was negative. Chest radiography indicated a cardiothoracic ratio of 58\% with pulmonary congestion and bilateral pleural effusions (Fig. 1A). Chest computed tomography revealed no bilateral hilar lymphadenopathy. Electrocardiography re- 
(A) Chest Xp

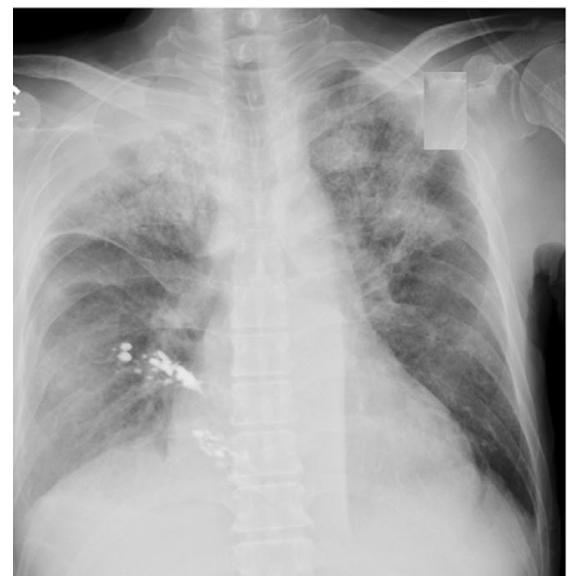

(B) Electrocardiogram

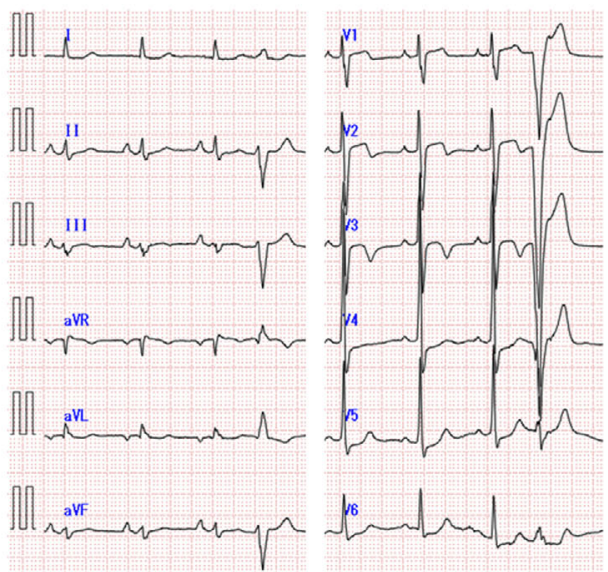

Figure 1. (A) Chest radiography performed on admission indicated a cardiothoracic ratio of $58 \%$ with pulmonary congestion and bilateral pleural effusions. (Note: calcification of the right hilar area resulting from previous aspiration of barium). (B) Electrocardiography performed on admission revealed a normal sinus rhythm, right bundle-branch block, premature ventricular contractions, and inverted $T$ waves in $\mathrm{V}_{2}-\mathrm{V}_{3}$.

\section{(A) On Admission}

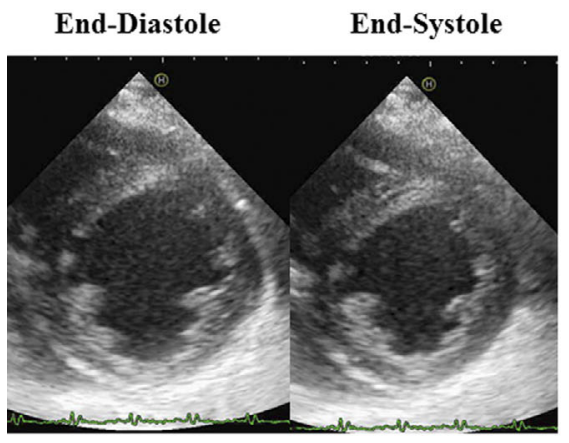

LV End-Diastolic Diameter: $53 \mathrm{~mm}$ LV End-Systolic Diameter: $48 \mathrm{~mm}$ LV Ejection Fraction: $25 \%$

MR: Moderate TR-PG: 32 mmHg
(B) 5-month After Steroid Therapy

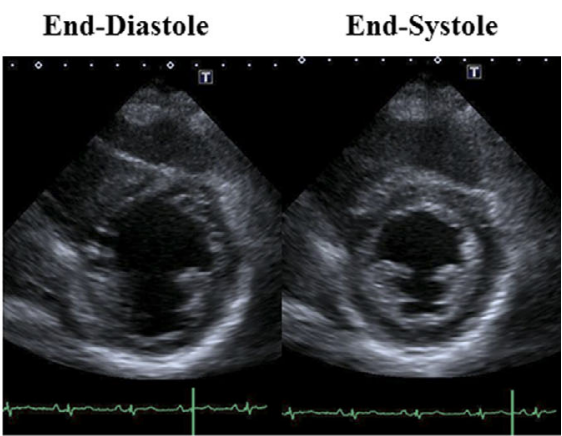

LV End-Diastolic Diameter: $51 \mathrm{~mm}$ LV End-Systolic Diameter: $35 \mathrm{~mm}$ LV Ejection Fraction: $50 \%$

MR: Mild

TR-PG: $15 \mathrm{mmHg}$

Figure 2. (A) Transthoracic echocardiography from the mid-left ventricular (LV) short axis view performed on admission showed that the $\mathrm{LV}$ ejection fraction (EF) calculated with the modified biplane Simpson's rule was $\mathbf{2 5 \%}$, and that the $\mathrm{LV}$ wall motion entailed severely diffuse hypokinesis. (B) Transthoracic echocardiography from the mid-LV short axis view performed five months after the initiation of steroid therapy demonstrated an improved LV systolic function with an LVEF of 50\%. MR: mitral regurgitation, TR: tricuspid regurgitation, PG: pressure gradient

vealed a normal sinus rhythm, right bundle-branch block, premature ventricular contractions, and inverted $\mathrm{T}$ waves in $\mathrm{V}_{2}-\mathrm{V}_{3}$ (Fig. 1B). A transthoracic echocardiographic examination disclosed that the LV ejection fraction (EF) calculated with the modified biplane Simpson's rule was $25 \%$, the LV end-diastolic diameter was $53 \mathrm{~mm}$ and the LV end systolic diameter was $48 \mathrm{~mm}$ (Fig. 2A). The LV wall motion consisted of severely diffuse hypokinesis with moderate mitral regurgitation. Moderate tricuspid regurgitation was also evident and the estimated pressure difference between the right ventricle and the atrium was $32 \mathrm{mmHg}$, which indicated the presence of pulmonary hypertension. Coronary angiography did not show significant stenosis, and the pulmonary capillary wedge pressure was $14 \mathrm{mmHg}$, the pulmonary artery pressure $31 / 15 \mathrm{mmHg}$, and the cardiac index was $1.69 \mathrm{~L} /$ $\mathrm{min} / \mathrm{m}^{2}$. Our initial diagnosis on admission was acute HF associated with acute myocarditis based on the detection of dilated cardiomyopathy. The patient was treated with oxygen inhalation, diuretics, dopamine, dobutamine and human atrial natriuretic peptide. Although the patient's clinical con- 


\section{(A) CMR}

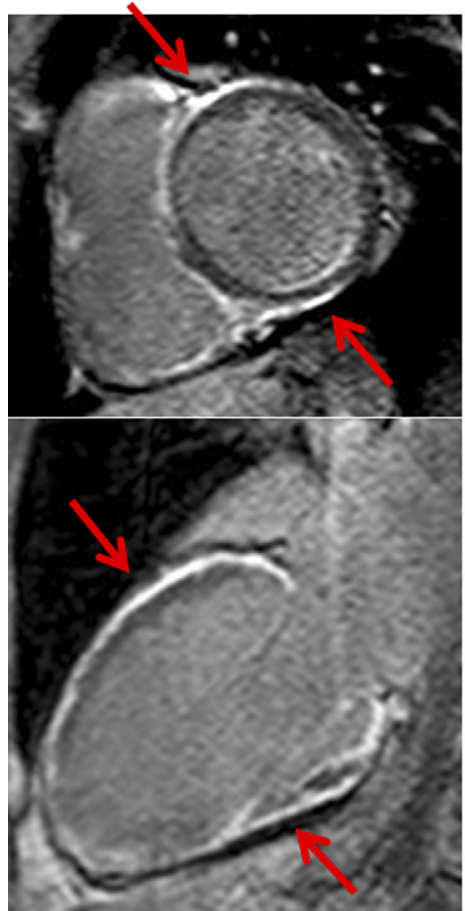

(B) FDG-PET

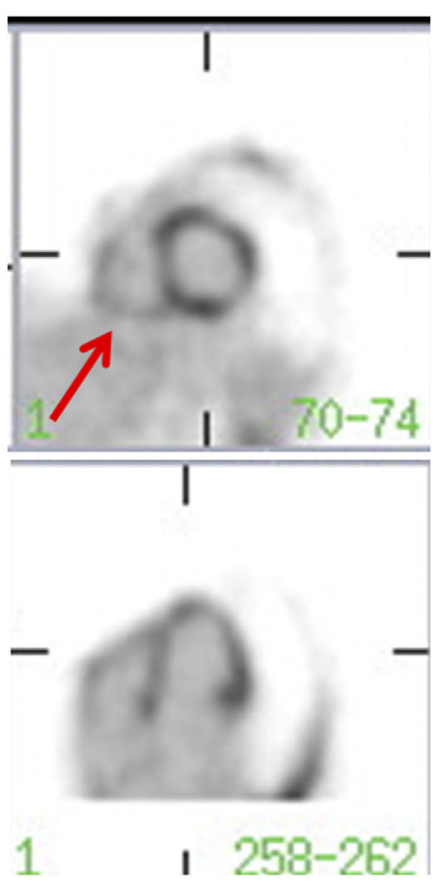

(C) Histologic findings of RV Endocardium

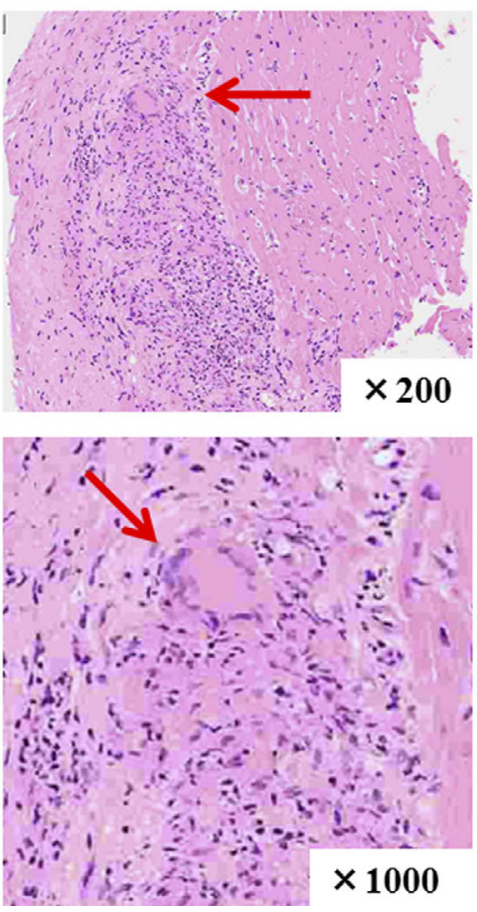

Figure 3. (A) Cardiac magnetic resonance imaging (CMR) disclosed late enhancement in the endocardial portion of the left ventricular (LV) septum and the inferior and right ventricles (arrow), however, no evidence of fatty infiltration or definite focal wall thinning was observed. (B) 18F-fluorodeoxy glucose (FDG)-positron emission tomography (PET) imaging showed significant increase in uptake by the LV septum and the lateral and right ventricular (RV) walls, indicating the presence of active inflammation (arrow). (C) The histologic findings of the endomyocardial biopsy specimens obtained from the right ventricle were indicative of epithelioid cell granuloma (arrow). The granulomas were non-necrotizing and composed of histiocytes, and lymphocytes, which is consistent with the presence of sarcoidosis.

dition continued to deteriorate, we felt that further assessment of acute HF due to LV dysfunction was required. Cardiac magnetic resonance imaging revealed severely diffuse LV systolic dysfunction with late enhancement in the endocardial portion of the LV septum and in the inferior and right ventricles, however, no evidence of fatty infiltration or definite focal wall thinning was observed (Fig. 3A). 18Ffluorodeoxy glucose-positron emission tomography imaging showed significantly increased patchy uptake by the LV septum and the lateral, and right ventricular walls, indicating the presence of active inflammation (Fig. 3B). The histologic findings of the endomyocardial biopsy specimens obtained from the right ventricle were indicative of epithelioid cell granuloma (Fig. 3C). The granulomas were nonnecrotizing and composed of histiocytes, and lymphocytes, findings that are consistent with the presence of sarcoidosis. The final diagnosis was thus acute HF caused by cardiac sarcoidosis with acute and active inflammation (1). No evidence of extra-cardiac sarcoidosis involving the lungs, lymph nodes, skin, liver or eyes was observed.

The patient was treated with prednisolone (40 mg/day), and his symptoms dramatically improved. Two months after the initiation of steroid therapy, the fluorodeoxy glucose (FDG) uptake had significantly decreased. Furthermore, five-months after the initiation of steroid therapy, the patient remained in New York Heart Association functional class I, however, transthoracic echocardiographic examination revealed a left ventricular ejection fraction (LVEF) of $50 \%$ and mild mitral regurgitation (Fig. 2B). Mild tricuspid regurgitation was also evident and the estimated pressure difference between the right ventricle and the atrium was 15 $\mathrm{mmHg}$.

\section{Discussion}

Sarcoidosis is a systemic granulomatous disease of undefined cause involving multiple organs, including the lungs, lymph nodes, eyes, heart and skin. Infiltration and resulting damage of the myocardium are important causes of death (2). Although autopsy studies have established that cardiac involvement is demonstrated in $20 \%$ to $50 \%$ of cases, cardiac sarcoidosis is symptomatic in only $5 \%$ of pa- 
tients, is an independent predictor of mortality and carries a very poor prognosis $(3,4)$. Although cardiac sarcoidosis entails significant morbidity and mortality due to the development of fatal arrhythmia, atrioventricular conduction disturbances, and refractory congestive heart failure, it is not always easy to diagnose. In addition, the patient in our case was diagnosed with cardiac sarcoidosis, however, it was difficult to distinguish cardiac sarcoidosis from giant cell myocarditis. The results of the Multicenter Idiopathic Giant Cell Myocarditis Registry suggest that cardiac sarcoidosis and idiopathic giant cell myocarditis are distinct clinicopathologic entities (5). The registry showed that, despite high rates of heart block, HF, and tachyarrhythmias, survival among patients with cardiac sarcoidosis is much better than that among patients with idiopathic giant cell myocarditis. Furthermore, a multivariate analysis revealed that presentation with HF predicts a diagnosis of idiopathic giant cell myocarditis, while presentation with heart block or more than nine weeks of symptoms predicts a diagnosis of cardiac sarcoidosis.

Uneven wall motion abnormalities characterized by the presence of dyskinetic or akinetic segments coexistent with normokinetic segments have been frequently observed in patients with cardiac sarcoidosis, a pattern which has also been documented in patients with myocarditis. The combination of granulomatous infiltration and scar formation with patchy distribution in the myocardium observed in patients with cardiac sarcoidosis is probably related to uneven LV wall motion. On the other hand, our patient developed acute HF due to severely diffuse LV wall motion abnormalities, however, such mimicking of acute myocarditis in the development of cardiac sarcoidosis is extremely rare. To the best of our knowledge, only a few similar cases have so far been previously reported (6-8). Our patients responded well to steroid therapy for cardiac sarcoidosis with acute inflammation rather than to standard pharmacological therapy for acute HF. Steroid therapy may be protective or therapeutic for preventing LV remodeling and preservating the LV function in the early or middle stages of cardiac sarcoidosis, however, it may not be as effective in the late stage. Early diagnosis and appropriate management of patients with cardiac sarcoidosis are thus essential. Moreover, sarcoid infiltration often occurs in the middle or epicardial portion of the LV wall in the early stage $(9,10)$, and findings on cardiac magnetic resonance imaging may reflect such a phenomenon.

In conclusion, the clinical history presented here suggests taht acute inflammation caused by sarcoidosis can lead to acute HF with severe LV dysfunction mimicking acute myocarditis. It is therefore important to identify this type of cardiac sarcoidosis at an early stage, when it can still successfully respond to steroid treatment.

The authors state that they have no Conflict of Interest (COI).

\section{References}

1. Guidelines for diagnosis and treatment of myocarditis (JCS 2009). Circ J 75: 734-743, 2011.

2. Iannuzzi MC, Rybicki BA, Teirstein AS. Sarcoidosis. New Engl J Med 357: 2153-2165, 2007.

3. Newman LS, Rose CS, Maier LA. Sarcoidosis. New Engl J Med 336: 1224-1234, 1997.

4. Deng JC, Baughman RP, Lynch JP 3rd. Cardiac involvement in sarcoidosis. Semin Respir Crit Care Med 23: 513-527, 2002.

5. Okura Y, Dec GW, Hare JM, et al. A clinical and histopathologic comparison of cardiac sarcoidosis and idiopathic giant cell myocarditis. Am Coll Cardiol 41: 322-329, 2003.

6. Chau EM, Fan KY, Chow WH. Cardiac sarcoidosis: a potentially fatal but treatable form of infiltrative heart disease. Hong Kong Med J 12: 65-67, 2006.

7. Miller A, Jackler I, Chuang M. Onset of sarcoidosis with left ventricular failure and multisystem involvement. Chest 70: 302-304, 1976.

8. Lopez JA, Hogan PJ, Fish RD, Capek P, Perin EC. Cardiac sarcoidosis. An unusual form of acute congestive cardiomyopathy. Tex Heart Inst J 22: 265-267, 1995.

9. Cheong BY, Muthupillai R, Nemeth $M$, et al. The utility of delayed-enhancement magnetic resonance imaging for identifying nonischemic myocardial fibrosis in asymptomatic patients with biopsy-proven systemic sarcoidosis. Sarcoidosis Vasc Diffuse Lung Dis 26: 39-46, 2009.

10. Kim JS, Judson MA, Donnino R, et al. Cardiac sarcoidosis. Am Heart J 157: 9-21, 2009.

(C) 2013 The Japanese Society of Internal Medicine http://www.naika.or.jp/imonline/index.html 